\title{
Analysis of vehicle-pedestrian and bicyclist conflicts in Győr-Hungary using Swedish conflict technique
}

\author{
A. Kizawi* \\ Széchenyi István University, Department of Transport Infrastructure and \\ Water Resources Engineering, Egyetem tér 1, 9026 Győr, Hungary \\ *e-mail: ahmad.kizawi.24@gmail.com
}

Submitted: 15/03/2021; Accepted: 26/04/2021; Published online: 18/05/2021

Abstract: Increasing traffic volumes leads to changes in traffic conditions, especially at intersections. These changes affect the ability of Vulnerable Road Users (VRUs) such as pedestrians and bicyclists to cross the road safely, especially at un-signalized crossings (without traffic lights), where many road users are conflicting at the same point of the road and neglecting 'in some cases' the priority issue. Although the emphasis on pedestrian safety has recently increased, there is still a need to analyze the causal factors of VRUs accidents and define their relationship to road design characteristics. This paper presents a study bout vehicle - pedestrian and bicyclist conflicts analysis using Swedish conflict technique at three un-signalized pedestrian crossings in the city of Györ- Hungary, where some pedestrian accidents were happened between 2014 and 2018, and reported based on accidents database in Hungary. The aim is to analyse vehicle-pedestrian and bicyclist interactions, and evaluate the severity of conflicts with the help of the Swedish technique graph. The findings concluded that there is a compatibility between Swedish conflict technique and accident records with regard to conflicts severity and the probability of accidents occurrence.

Keywords: traffic conflict; Swedish conflict technique; Vulnerable Road Users (VRUs) safety 


\section{Introduction}

Road accidents is a major cause of injury and death, especially to pedestrians. Not all pedestrians have the same ability to cross the road easily. Many have some restrictions that require special attention on their part and/or modifications of roadway infrastructure and operations to accommodate their needs. [1] The unsignalized intersection that is not controlled by traffic lights is a popular situation in any road network, as it is used by two or more traffic flows, which have different priorities. At un-signalized intersections, the absence of traffic lights may disregard, in some cases, the priority of vulnerable road-users to cross, such as pedestrians and bicyclists, or restrict their ability to pass safely. [2]

The emphasis on the safety of VRUs has recently received more interest. Since the 1960 's, several traffic conflict studies have been accomplished aiming to develop new methods to evaluate road safety issues in different road geometric and operating conditions. [3] Traffic Conflict Techniques (TCTs) can be used as alternative or complementary tools for the use of accident records to verify traffic conflicts and estimate accidents probability [4]. Traffic conflict indicators are applicable to assess road safety performance thanks to their ability to capture conflict data, including the severity of conflicts, in an objective way within a shorter time compared to accident records.

One of the most common conflict techniques is the Swedish technique; it is based on observing the traffic conflicts between two road-users, with their evasive actions taken to avoid collision. The main characteristics of this technique are related to several points such as the requirement for a collision course in a conflict; the conflict severity based on the onset of an evasive action; and the distinction between severe and non-severe conflicts. The serious conflicts were found to be an indicator of a breakdown in the interaction - similar to a breakdown preceding an accident. [5]

This technique aims to analyze traffic conflicts on a given road, where the probability of accident occurrence is highly expected. This technical approach provides a better insight into conflict characteristics and helps in estimating the severity of conflicts and providing a possibility to suggest accidents countermeasures to improve the safety of VRUs. 


\section{Problem statement}

Many pedestrians (especially from children and elderly people) find it difficult and unsafe to cross at un-signalized crossings that are not controlled by traffic lights. [6]. At pedestrian crossings that are not controlled by pedestrian traffic lights (unsignalized crosswalks), the priority of the pedestrian is not clear, even with legislation supporting pedestrian priority, and this results in higher levels of accident and death of pedestrians compared to those controlled by pedestrian traffic lights. Moreover, pedestrians often tend to make more sudden decisions and more prone to hectically change their speed and trajectory. Nowadays, it is obvious that the traffic conflict studies at un-signalized intersections are quite necessary. Many researchers have focused on this approach aiming at improving this situation. [7]. An accident analysis has been accomplished in order to determine the main causes of pedestrian related accidents based on the records of WEB-BAL (Hungarian accident database) from year 2018 [8]. Fig. 1 shows the results of the analysis. In $63 \%$ of all pedestrian related accidents, drivers are at fault while in $37 \%$ pedestrians mistaken. The highest share of causes is yield failure, but speeding and turning failure are also responsible for a high proportion of the pedestrian related crashes.

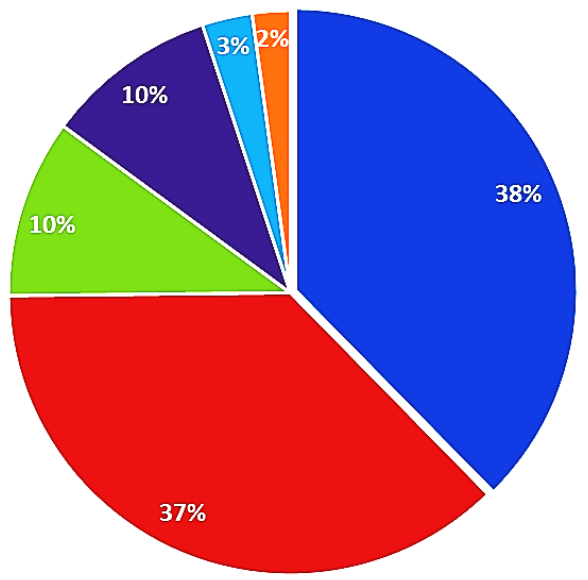

- Yield failure

- Pedestrians' fault

$\square$ Speeding

- Changeover, turning failure

口 Other drivers' fault

- Overtaking mistake

Figure 1. Causes of pedestrian accidents in Hungary, 2018 (based on [8])

Fig. 2 shows the results of the accident type analysis in Hungary 2018. Pedestrian accidents occurring at designated pedestrian crossings in intersection have the highest share among the accident types. (29\% of accidents are not classified.) 
A. Kizawi - Acta Technica Jaurinensis, Vol. 14, No. 4, pp. 377-405, 2021

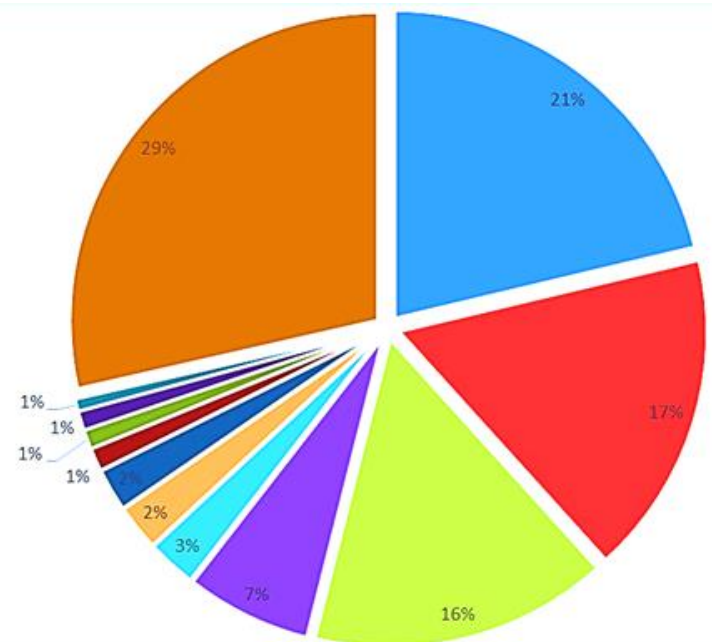

- Pedestrian accident at ped-crossing in intersection

- Pedestrian accident not in intersection

= Pedestrian accident at ped-crossing, not in intersection

- Pedestrian accident in intersection, not at ped-crossing

= Pedestrian accident not in intersection, walking in the opposite direction to automobile traffic

= Pedestrian accident outside the roadway, on the RIGHT hand side (on sidewalk, bus stop, etc.)

- Pedestrian accident, crossing in front of / behind a standing vehicle or other obstacle on the right hand side of the road

- Pedestrian accident on the roadway, at a bus/tram stop

a Pedestrian accident outside the roadway, on the LEFT hand side (on sidewalk, bus stop, etc.)

- Pedestrian accident NOT in intersection, in a ped-crossing, passing a standing or moving vehicle

a Pedestrian accident in intersection, at ped-crossing, passing a standing car

- Other type of pedestrian accident

Figure 2. Pedestrian accidents according to accident type in Hungary, 2018 (based on [8]) 


\section{Literature review}

The idea of the conflict had been around for many years before it was first carried out systematically in the traffic safety community in the late 1960s. The first practical application of a traffic conflict technique was accomplished in the late 1960s by a team of researchers at General Motors Corporation Perkins and Harris [9]. Since that time, traffic conflict techniques have been developed and used more frequently.

The first study to develop a measure by which accidents can be predicted was designed by Perkins and Harris [9]. More than twenty types of potential traffic conflicts were identified and defined by the occurrence of evasive actions, such as braking or sudden change the path. Since that time, and for many objectives, traffic conflict techniques have been developed in many countries, although in most cases the emphasis is still on the diagnosis of safety problems. [4]

The common governing indicator for all methods addressed of most literatures regarding traffic conflicts is "Time-to-Collision"(TTC), which defined as time remaining to collide for two conflicting road users if they continue on the same trajectory and at their present speed [4].

In the 1970's and 1980's, the traffic conflict research noted significant development at the Technical University of Lund, Sweden. The method of traffic conflict observation is carried out by trained observers focused on situations happen on the road, in which two road users would collide if neither of them made some kind of an evasive action, such as stopping, braking, or changing the lane. The point, in which such evasive action is made, is recorded personally by the observer as the "Time-to-Accident" (TA). The TA value, the speed of conflict, and the distance between the two conflicting road users are used to determine whether the conflict is severe or not using a graph, which has disaggregating lines to categories the different conflicts according to severity of the conflict. According to the country of origin, this method has been called the "Swedish Traffic Conflict Technique" [10]. Reliability tests indicate very strongly that observers record conflicts in a reliable way after some days of training. The results from validation studies indicate that this technique, based on a criterion that the degree of seriousness should be taken into account, shows a strong correlation between conflicts and accidents. [11].

According to the literature review provided by A. Kizawi et al. on the conflict analysis of vehicle-pedestrian interactions, the frequent interactions between vehicles and pedestrians deserve special interest to analyze safety especially at intersections. As an alternative to traffic safety analysis based on historical crash data the use of non-crash events is becoming more popular. Various researchers have developed a number of safety indicators (e.g. Time to Accident (TA), which is 
related to one of the most commonly used Surrogate Measures of Safety "Time-To Collision", where TA is calculated at the moment in which an involved road user makes an evasive action). There is a consensus among researches that observable non-crash events can be useful for traffic safety assessment as a substitutional tool in parallel with analysis based on crash data [12].

Almqvist and Hydén [13] [14] applied proximity based technique i.e. Swedish Traffic Conflict Technique (conflict observation using Time to Accident) in the city of Cochabamba in Bolivia with a view to guiding a method to assess the safety problem in developing countries. The study indicated that the technique is useful in its present form for this condition.

Tiwari et al. [15] evaluated conflicts at 14 locations in Delhi, in a heterogeneous traffic environment. The conflict was evaluated using the concept of Time-toaccident (TA). This study recorded seven types of conflicts occurring at mid-block in heterogeneous traffic, such as head-on, rear-end, sideswipe, change direction, fixed object, angle and traverse angle.

\section{Conflict observation using Swedish technique}

According to the Swedish Traffic Conflict Technique STCT, a collision course is a necessary condition for a traffic conflict. Collision course implies that, unless one of the road users takes an evasive action, a collision will occur. The severity of a conflict can be defined at the moment in which one of the road users takes an evasive action such as hard braking, sudden stopping, or sudden changing the lane. The relevant road user is the name of the road user who firstly takes an evasive action to avoid the collision with the other user. [10]

The conflict severity is based on two indicators: [10]

1. Time-to-Accident (TA): it is the time remaining to a collision when the relevant road user takes an evasive action. Lower TA values indicates that the traffic conflict is nearer to a collision, this means the situation is more dangerous.

2. Conflicting Speed (CS): it is the speed of the relevant road user when taking the evasive action to avoid the collision with the other road user. Higher CS values lead to a more severe conflict.

In practice, when this method is carried out at a given location personally, it would be easy to estimate the distance from the road user to the collision point rather than the time remaining to the collision. In this method, we estimate the distance between the two conflicting road users and the speed of the relevant road user. After that, the conversion table (see Table 1) can be used to calculate time to accident (TA) based on the distance to the collision point and the speed of the relevant road user. 
Table 1. Time-to-accident values based on conflict speed and distance (based on [10])

\begin{tabular}{|c|c|c|c|c|c|c|c|c|c|c|c|c|c|c|c|c|c|c|c|c|c|}
\hline \multicolumn{2}{|c|}{ Speed } & \multicolumn{20}{|c|}{ Distance [m] } \\
\hline$\underline{\underline{\Xi}}$ & $\stackrel{n}{\Xi}$ & $\ddot{o}$ & - & $N$ & $m$ & $\nabla$ & $n$ & 0 & $r$ & $\infty$ & $a$ & 으 & 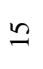 & ㄱ & $\approx$ & ల & $\stackrel{n}{n}$ & 우 & $\stackrel{\wp}{f}$ & ㄴ & $n$ \\
\hline$n$ & $\stackrel{+}{\sim}$ & $\stackrel{+}{0}$ & 5 & $\stackrel{+}{-}$ & $\widetilde{N}$ & $\widehat{\Omega}$ & $\ddot{\varphi}$ & $\stackrel{m}{\forall}$ & in. & $\begin{array}{l}\infty \\
n\end{array}$ & ?ִ & $\stackrel{\text { Nִ }}{n}$ & & & & & & & & & \\
\hline 으 & $\begin{array}{l}\infty \\
\sim \\
\sim\end{array}$ & Nִ & $\stackrel{+}{0}$ & $\tilde{0}$ & ב & $\stackrel{+}{\sim}$ & $\infty$ & $\stackrel{\sim}{\sim}$ & $\stackrel{n}{\sim}$ & $\hat{\imath}$ & $\stackrel{\sim}{m}$ & $\stackrel{\varphi}{r}$ & $\dot{v}$ & Nִ & $\stackrel{\circ}{\circ}$ & & & & & & \\
\hline$\because$ & $\stackrel{\sim}{+}$ & $\overrightarrow{0}$ & $\tilde{o}$ & ?ִ & $\tilde{o}$ & $\stackrel{0}{0}$ & $\stackrel{\text { Iִ }}{ }$ & $\stackrel{+}{\sim}$ & I. & 9 & $\underset{\sim}{\sim}$ & $\stackrel{+}{\sim}$ & $\begin{array}{l}\dot{\varphi} \\
\dot{\varphi}\end{array}$ & $\stackrel{\infty}{+}$ & $\stackrel{0}{0}$ & $\stackrel{n}{r}$ & $\underset{\infty}{+}$ & $\stackrel{0}{a}$ & & & \\
\hline$\stackrel{i}{\text { i }}$ & $\begin{array}{l} \\
\dot{n}\end{array}$ & $\overrightarrow{0}$ & $\tilde{o}$ & $\stackrel{\Delta}{0}$ & $\tilde{n}$ & $\tilde{0}$ & $\ddot{0}$ & 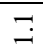 & $\dddot{m}$ & $\stackrel{+}{-}$ & $\stackrel{0}{-}$ & $\stackrel{\infty}{\infty}$ & $\vec{i}$ & $\stackrel{\varphi}{\varphi}$ & $\stackrel{n}{q}$ & $\stackrel{\nabla}{r}$ & $\tilde{b}$ & $\stackrel{n}{r}$ & $\vec{\infty}$ & $\stackrel{\circ}{\circ}$ & $\dot{\sigma}$ \\
\hline 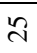 & $\hat{\theta}$ & $\overline{0}$ & $\overrightarrow{0}$ & $\tilde{0}$ & $\stackrel{\square}{0}$ & $\begin{array}{l}0 \\
0\end{array}$ & $\tilde{0}$ & $\ddot{0}$ & $\underset{-}{0}$ & $\stackrel{\text { I }}{-}$ & $\stackrel{n}{n}$ & $\stackrel{+}{-}$ & $\stackrel{\sim}{N}$ & $\hat{\sigma}$ & $\stackrel{\varphi}{\dot{m}}$ & $\stackrel{?}{+}$ & ị. & $\begin{array}{l}\infty \\
i \\
i\end{array}$ & $\tilde{q}$ & $\stackrel{N}{N}$ & $\stackrel{9}{\pi}$ \\
\hline in & $\stackrel{\infty}{\infty}$ & $\overrightarrow{0}$ & $\overrightarrow{0}$ & กั & ナ. & $\dddot{n}$ & $\ddot{0}$ & $\hat{o}$ & $\stackrel{\infty}{0}$ & $\stackrel{0}{\circ}$ & $=$ & $\stackrel{-1}{~}$ & $\stackrel{\infty}{\infty}$ & $\stackrel{i}{i}$ & 아. & $\ddot{l}$ & $\stackrel{+}{\stackrel{4}{*}}$ & $\stackrel{\infty}{+}$ & $\ddot{v}$ & $\stackrel{0}{0}$ & $\underset{0}{0}$ \\
\hline$m$ & $\hat{\sigma}$ & $\overrightarrow{0}$ & $\overrightarrow{0}$ & ֻै & ? & $\stackrel{+}{0}$ & $\dddot{n}$ & $\stackrel{0}{0}$ & $\tilde{0}$ & $\stackrel{\infty}{0}$ & 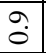 & $\stackrel{0}{0}$ & $\because$ & $\overrightarrow{\mathrm{i}}$ & $\begin{array}{l}\sigma \\
\text { i }\end{array}$ & $\vec{m}$ & $\begin{array}{l}\dot{\varphi} \\
\end{array}$ & F & $\begin{array}{l}\dot{\gamma} \\
\dot{\gamma}\end{array}$ & $\vec{n}$ & in \\
\hline 우 & 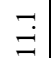 & $\stackrel{\circ}{0}$ & $\overrightarrow{0}$ & ํ. & $\tilde{0}$ & $\stackrel{+}{0}$ & $\dddot{n}$ & $\tilde{n}$ & $\stackrel{0}{0}$ & $\hat{0}$ & $\stackrel{\infty}{0}$ & $\ddot{0}$ & $\stackrel{+}{\sim}$ & $\stackrel{\infty}{\rightarrow}$ & $\stackrel{m}{\sim}$ & $\tilde{\sim}$ & rே & $\stackrel{b}{\dot{m}}$ & $\exists$ & $\stackrel{n}{\forall}$ & $\stackrel{\circ}{i}$ \\
\hline 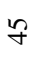 & $\stackrel{\sim}{\simeq}$ & & $\ddot{0}$ & $\stackrel{\sim}{0}$ & ஸ̣ & $\ddot{0}$ & $\stackrel{+}{0}$ & $\tilde{n}$ & $\stackrel{0}{0}$ & $\stackrel{0}{0}$ & $\hat{0}$ & $\stackrel{\infty}{0}$ & $\stackrel{\text { Iִ }}{ }$ & $\stackrel{0}{\circ}$ & $\stackrel{\circ}{i}$ & $\stackrel{+}{\sim}$ & $\stackrel{\infty}{i}$ & $\stackrel{n}{m}$ & $\stackrel{\circ}{\dot{m}}$ & $\stackrel{\circ}{+}$ & $\stackrel{+}{+}$ \\
\hline in & $\ddot{g}$ & & $\overline{0}$ & $\overrightarrow{0}$ & ִָ & $\stackrel{m}{0}$ & $\stackrel{+}{0}$ & $\stackrel{+}{0}$ & n? & $\stackrel{0}{0}$ & $\stackrel{0}{0}$ & ڤ. & 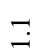 & $\stackrel{+}{\sim}$ & $\stackrel{\infty}{\infty}$ & 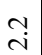 & $\underset{n}{n}$ & $\stackrel{\hat{i}}{ }$ & $\stackrel{n}{n}$ & $\ddot{m}$ & $\stackrel{\circ}{+}$ \\
\hline$n$ & m & & $\overrightarrow{0}$ & $\overrightarrow{0}$ & ִָ & $\stackrel{m}{0}$ & $\dddot{m}$ & $\stackrel{+}{0}$ & 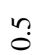 & 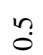 & $\stackrel{\bullet}{0}$ & $\hat{\sigma}$ & 움. & $\dddot{m}$ & $\stackrel{\circ}{\circ}$ & $\stackrel{\circ}{i}$ & $\stackrel{n}{n}$ & $\underset{i}{\stackrel{0}{i}}$ & $\hat{\mathrm{i}}$ & $\stackrel{m}{m}$ & $\stackrel{\circ}{\ddot{r}}$ \\
\hline 8 & $\ddot{\theta}$ & & $\overline{0}$ & $\overrightarrow{0}$ & ִָ & ํ. & $\ddot{0}$ & $\stackrel{+}{0}$ & $\stackrel{+}{0}$ & $\tilde{0}$ & $\dddot{n}$ & $\begin{array}{l}0 \\
0\end{array}$ & $\ddot{0}$ & $\stackrel{\text { Iִ }}{\longrightarrow}$ & $\because$ & $\stackrel{\infty}{-}$ & $\overrightarrow{\mathrm{i}}$ & $\stackrel{+}{\sim}$ & $\stackrel{\sim}{\sim}$ & $\underset{\text { ஸे }}{\stackrel{0}{ }}$ & $\stackrel{m}{m}$ \\
\hline$\sqrt[6]{6}$ & $\vec{\infty}$ & & $\ddot{0}$ & 3 & ָ̦ & $\tilde{0}$ & $\tilde{0}$ & ? & ț & $\stackrel{t}{0}$ & 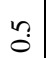 & $\stackrel{0}{0}$ & $\stackrel{\infty}{0}$ & $\Xi$ & $\stackrel{+}{\dot{H}}$ & I & $\stackrel{9}{2}$ & ָָ & $\tilde{n}$ & $\stackrel{\infty}{i}$ & $\stackrel{\circ}{m}$ \\
\hline & $\stackrel{\nabla}{\Delta}$ & & $\overrightarrow{0}$ & $\overrightarrow{0}$ & $\tilde{o}$ & ָั & $\stackrel{m}{0}$ & $\stackrel{m}{0}$ & $\dot{0}$ & $\stackrel{+}{0}$ & $\because$ & $\because$ & $\stackrel{\infty}{0}$ & 움. & $\stackrel{m}{q}$ & $\dddot{n}$ & $\stackrel{\infty}{\sim}$ & $\vec{c}$ & $\ddot{n}$ & $\stackrel{o}{i}$ & $\stackrel{\infty}{i}$ \\
\hline$\stackrel{n}{n}$ & $\stackrel{\infty}{\stackrel{0}{0}}$ & & $\stackrel{\circ}{0}$ & $\overrightarrow{0}$ & $\overrightarrow{0}$ & $\stackrel{1}{0}$ & $\tilde{O}$ & $\stackrel{m}{0}$ & $\stackrel{m}{0}$ & $\stackrel{t}{0}$ & ப் & $\ddot{0}$ & $\tilde{0}$ & 움. & 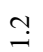 & $\stackrel{+}{\dot{Z}}$ & $\cong$ & 9 & $\underset{n}{n}$ & $\stackrel{i}{i}$ & $\underset{i}{i}$ \\
\hline$\infty$ & ָָ & & $\stackrel{\circ}{0}$ & $\overrightarrow{0}$ & $\overrightarrow{0}$ & ํ. & ํ. & m? & $\tilde{0}$ & $\stackrel{+}{0}$ & $\stackrel{+}{0}$ & $\tilde{n}$ & $\tilde{0}$ & $\stackrel{0}{0}$ & $\rightrightarrows$ & $\stackrel{+}{\sim}$ & $\stackrel{0}{0}$ & $\stackrel{\infty}{\infty}$ & $\stackrel{\circ}{i}$ & $\stackrel{m}{\sim}$ & $\stackrel{n}{i}$ \\
\hline 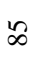 & $\begin{array}{l}\dot{\theta} \\
\ddot{\lambda}\end{array}$ & & $\stackrel{\circ}{0}$ & $\overrightarrow{0}$ & $\overrightarrow{0}$ & $\stackrel{\Upsilon}{0}$ & $\stackrel{N}{0}$ & $\stackrel{m}{0}$ & $\stackrel{n}{0}$ & $\stackrel{m}{0}$ & $\stackrel{+}{0}$ & $\stackrel{+}{\circ}$ & $\stackrel{0}{0}$ & $\stackrel{\infty}{0}$ & $\rightrightarrows$ & $\dddot{m}$ & $\because$ & f. & $\stackrel{9}{-}$ & $\vec{i}$ & $\stackrel{m}{\sim}$ \\
\hline ஃ & $\begin{array}{l}0 \\
\ddot{\sim}\end{array}$ & & $\stackrel{\circ}{0}$ & $\overrightarrow{0}$ & $\overrightarrow{0}$ & $\stackrel{1}{0}$ & $\stackrel{1}{0}$ & ִָ & $\stackrel{n}{0}$ & ?ִ & $\stackrel{+}{0}$ & $\stackrel{+}{0}$ & $\stackrel{0}{0}$ & $\stackrel{\infty}{0}$ & $\stackrel{0}{-}$ & $\stackrel{\text { Iִ }}{\longrightarrow}$ & $\stackrel{+}{+}$ & $\stackrel{0}{-}$ & $\stackrel{\infty}{-}$ & $\stackrel{\circ}{i}$ & $\stackrel{\sim}{i}$ \\
\hline$\approx$ & ષ্ণ & & $\stackrel{0}{0}$ & $\overrightarrow{0}$ & $\ddot{0}$ & กָ & ํํ & ํ. & $\stackrel{m}{0}$ & $\stackrel{n}{0}$ & $\stackrel{m}{0}$ & $\stackrel{+}{\circ}$ & $\stackrel{\bullet}{0}$ & $\stackrel{\infty}{0}$ & $\ddot{\sigma}$ & 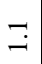 & $\dddot{m}$ & $\because$ & $\exists$ & 9 & $\vec{i}$ \\
\hline 8 & $\stackrel{\infty}{\grave{N}}$ & & $\stackrel{\circ}{\circ}$ & $\overrightarrow{0}$ & $\overrightarrow{0}$ & $\overrightarrow{0}$ & ํ. & $\stackrel{\sim}{0}$ & $\dddot{m}$ & ? & $\stackrel{m}{0}$ & $\stackrel{t}{\circ}$ & $\because$ & $\tilde{0}$ & 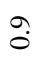 & $\leftrightarrows$ & $\stackrel{m}{9}$ & $\stackrel{+}{-}$ & بִ & $\stackrel{\infty}{\longrightarrow}$ & $\stackrel{\circ}{i}$ \\
\hline
\end{tabular}


The graph shown in Fig. 3 can be used to define the severity level of the traffic conflict based on the TA and CS values. [10] According to the graph, the conflicts with severity level above 26 (red line on the graph) are ranked as serious, while the conflicts with severity under 26 are ranked as non-serious conflicts. The serious conflicts have a strong statistical relation with police-reported accidents and even can be converted into expected number of accidents with a reasonable accuracy. [10]

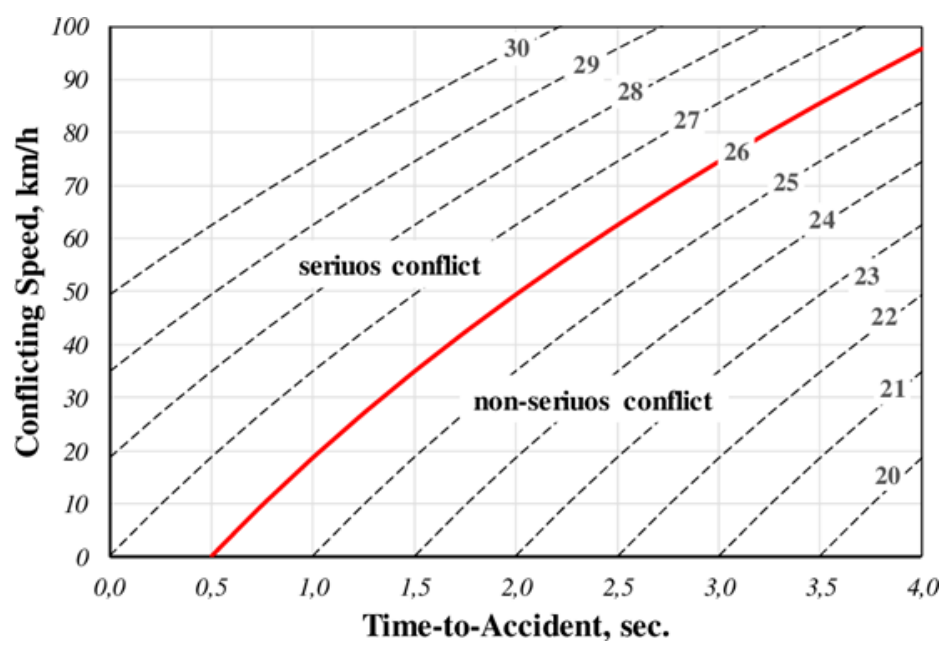

Figure 3. Swedish technique graph [10]

\section{Methodology}

\subsection{Sites selection}

The pedestrian accident data that observed in the city of Györ between 2014 and 2018 have been signed on a map using google map service [16] as shown in Fig 4. This map contains locations where accidents were occurred and reported with details; (accident location, accident date, causes of the accident and other relevant information). The accidents were distinguished by colours according to their type as follows:

- Pedestrian accident, crossing in front of / behind a standing vehicle or other obstacle on the right hand side of the road

- Pedestrian accident, vehicle hitting the pedestrian outside the road on the LEFT hand side (e.g. on the sidewalk)

- Pedestrian accident, vehicle hitting the pedestrian outside the road on the RIGHT hand side (e.g. on the sidewalk) 
- Pedestrian accident, vehicle hitting the pedestrian at a bus (or other PT) stop

- Pedestrian accident NOT in intersection, in a pedestrian-crossing, passing a standing or moving vehicle

- Pedestrian accident at pedestrian crossing, not in intersection

- Pedestrian accident in intersection, at pedestrian crossing

- Pedestrian accident in intersection, at pedestrian crossing, passing a standing car

- Pedestrian accident not in intersection, walking in the opposite direction to automobile traffic

- Pedestrian accident in intersection, not a pedestrian crossing, passing a standing car

- Pedestrian accident not in intersection

- Irregular pedestrian movement at the designated pedestrian crossing

- Other type of pedestrian accident

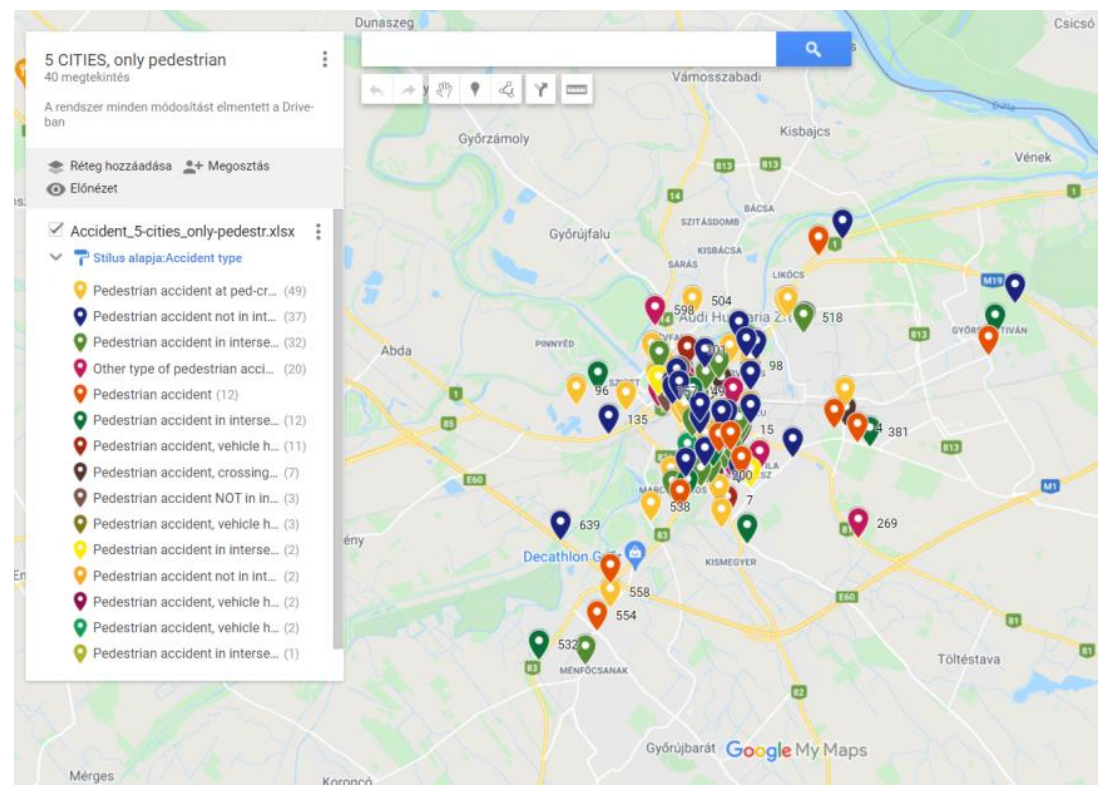

Figure 4. Pedestrian accidents map in Györ city - Hungary (based on [8])

According to the accident map, pedestrian accidents are attributed to different causes at different road characteristics. After trying to figure out a common denominator between many locations by this map to deal with a specific situation three locations could be selected with the same road type (un-signalized intersection 
with three legs, with only one pedestrian crossing on the main road), where at least an accident happened at the pedestrian crossing.

\subsection{Sites visit}

Before starting the conflicts observation, each location was personally visited. Two hours, at least, were spent for the first visit for each location. The aim was to recognize the road users who are frequently present and the prevailing traffic conflicts at each location.

\subsection{Sites description}

\subsubsection{Location (1): Tihanyi Árpád út 73, 9023 - Kassák Lajos 73 St}

This location is the intersection of the main road (Tihanyi Árpád út 73, 9023) and the secondary road (Kassák Lajos 73 St) (see Figs. 5 and 6). A zebra crossing exists on the main road. This location is one of the most crowded place in the city, where all categories of road-users exist (vehicles, buses, motorcycles, bicyclists, and pedestrians). Many facilities exist near this intersection such as Fekete István Győr Primary School, Petz Aladár County Teaching Hospital, and Győr Pláza shopping Mall. Table 2 shows some details related to this location. The specifications of recorded accidents are listed in Table.3.

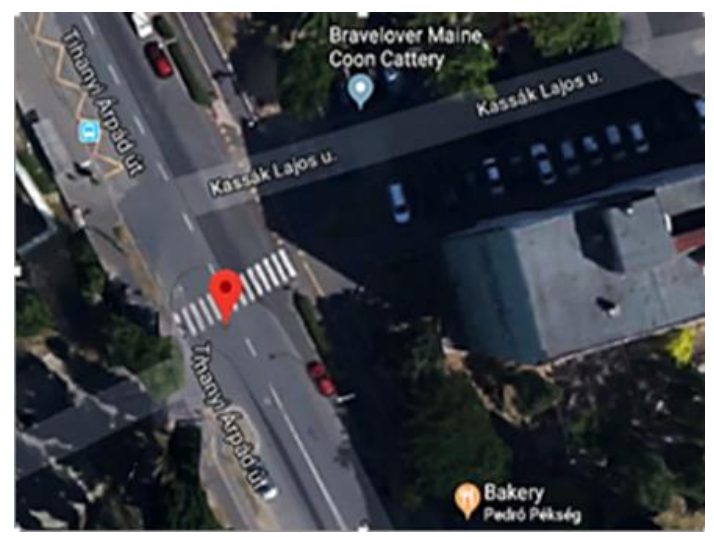

Figure 5. Tihanyi Árpád út 73, 9023 - Kassák Lajos 73 St. [16] 
Table 2. Some details related to location (1) (Tihanyi Árpád út 73, 9023 - Kassák Lajos 73 St.) (based on [8] and [16])

\begin{tabular}{|c|c|c|c|}
\hline City & The main road & GPS Coordinates & $\begin{array}{c}\text { Number of } \\
\text { accidents observed } \\
\text { according to } \\
\text { accidents map }\end{array}$ \\
\hline Győr & Tihanyi Árpád út & $47^{\circ} 40^{\prime} 19.8^{\prime \prime} \mathrm{N}$ & 3 \\
& 73,9023 & $17^{\circ} 39^{\prime} 03.6^{\prime \prime} \mathrm{E}$ & \\
\hline
\end{tabular}

Table 3. The specifications of recorded accidents at location (1) (Tihanyi Árpád út 73, 9023 - Kassák Lajos 73 St.) (based on [8] and [16])

\begin{tabular}{|c|c|c|c|c|}
\hline & $\begin{array}{c}\text { Time of } \\
\text { accident }\end{array}$ & $\begin{array}{c}\text { Outcome } \\
\text { of } \\
\text { accident }\end{array}$ & $\begin{array}{c}\text { GPS } \\
\text { Coordinates }\end{array}$ & Accident Type \\
\hline $\begin{array}{c}\text { Accident } \\
1\end{array}$ & $19 / 4 / 2018$ & $\begin{array}{c}\text { Slight } \\
\text { injury }\end{array}$ & $\begin{array}{c}47.672166 \\
17.650999\end{array}$ & $\begin{array}{c}\text { irregular movement } \\
\text { at the designated } \\
\text { pedestrian crossing }\end{array}$ \\
\hline $\begin{array}{c}\text { Accident } \\
2\end{array}$ & $8 / 1 / 2016$ & $\begin{array}{c}\text { Slight } \\
\text { injury }\end{array}$ & $\begin{array}{c}47.672194 \\
\text { irregular movement } \\
\text { at the designated } \\
\text { pedestrian crossing }\end{array}$ \\
\hline $\begin{array}{c}\text { Accident } \\
3\end{array}$ & $13 / 12 / 2017$ & $\begin{array}{c}\text { Slight } \\
\text { injury }\end{array}$ & 47.672194 & $\begin{array}{c}\text { irregular movement } \\
\text { at the designated } \\
\text { pedestrian crossing } \\
\text { with another vehicle } \\
\text { (standing) }\end{array}$ \\
\hline
\end{tabular}




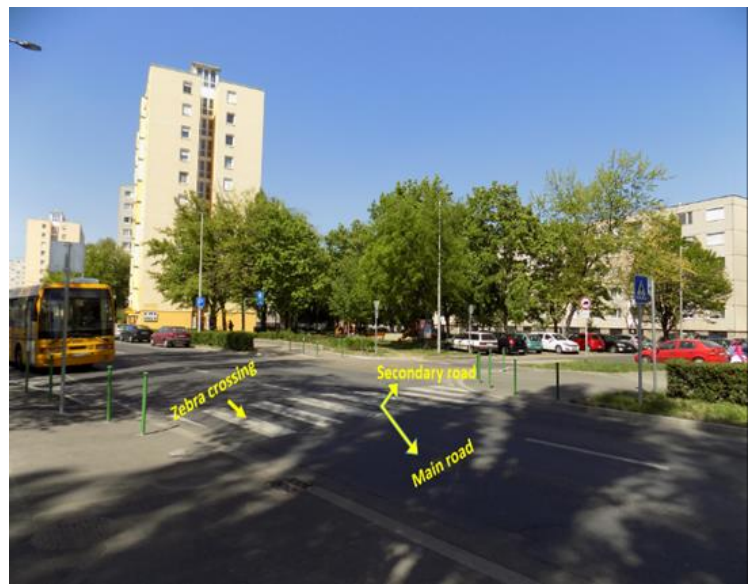

Figure 6. Tihanyi Árpád út 73, 9023 - Kassák Lajos 73 St.

\subsubsection{Location (2): Tihanyi Árpád út 78, 9023 way - Jereváni way}

This location is the intersection of the main road (Tihanyi Árpád út 78, 9023 way -Jereváni way) and the secondary road (to Győr Pláza shopping Mall) (see Figs. 7 and 8). A zebra crossing exists on the main road. This location is also one of the most crowded place in the city. This location exists very close to location (1). Table 4 shows some details related to this location. The specifications of recorded accidents are listed in Table 5.

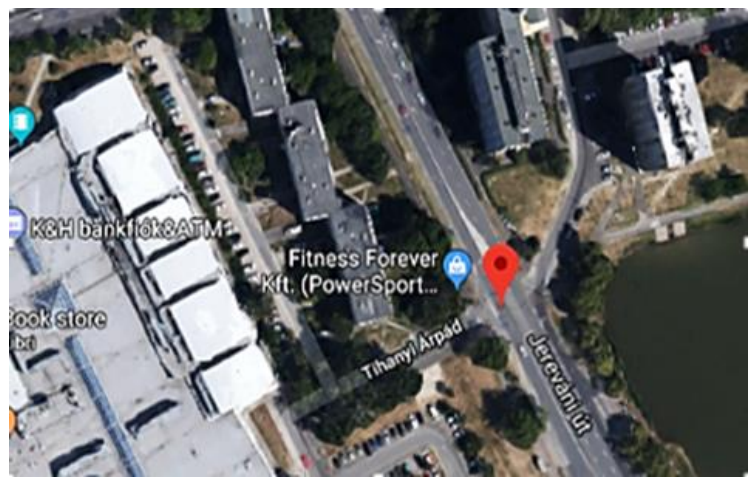

Figure 7. Tihanyi Árpád út 78, 9023 way - Jereváni way [16] 
Table 4. Some details related to location (2) (Tihanyi Árpád út 78, 9023 way - Jereváni way) (based on [8])

\begin{tabular}{|c|c|c|c|}
\hline City & The main road & GPS Coordinates & $\begin{array}{c}\text { Number of } \\
\text { accidents } \\
\text { observed } \\
\text { according } \text { to } \\
\text { accidents map }\end{array}$ \\
\hline Györ & Tihanyi Árpád út 78, & $47^{\circ} 40^{\prime} 11.3^{\prime \prime} \mathrm{N}$ & 2 \\
& 9023 & $17^{\circ} 39^{\prime} 10.1^{\prime \prime} \mathrm{E}$ & \\
\hline
\end{tabular}

Table 5. The specifications of recorded accidents at location (2) (Tihanyi Arpád út 78, 9023 way - Jereváni way) (based on [8] and [16])

\begin{tabular}{|c|c|c|c|c|}
\hline & $\begin{array}{c}\text { Time of } \\
\text { accident }\end{array}$ & $\begin{array}{c}\text { Outcome } \\
\text { of } \\
\text { accident }\end{array}$ & GPS & Accident Type \\
\hline $\begin{array}{c}\text { Accident } \\
1\end{array}$ & $14 / 11 / 2015$ & $\begin{array}{c}\text { serious } \\
\text { injury }\end{array}$ & $\begin{array}{c}47.669805 \\
17.652805\end{array}$ & $\begin{array}{c}\text { irregular movement } \\
\text { at the designated } \\
\text { pedestrian crossing }\end{array}$ \\
\hline $\begin{array}{c}\text { Accident } \\
2\end{array}$ & $6 / 2 / 2017$ & $\begin{array}{c}\text { Slight } \\
\text { injury }\end{array}$ & $\begin{array}{l}47.669916 \\
17.652666\end{array}$ & $\begin{array}{c}\text { irregular movement } \\
\text { at the designated } \\
\text { pedestrian crossing }\end{array}$ \\
\hline
\end{tabular}




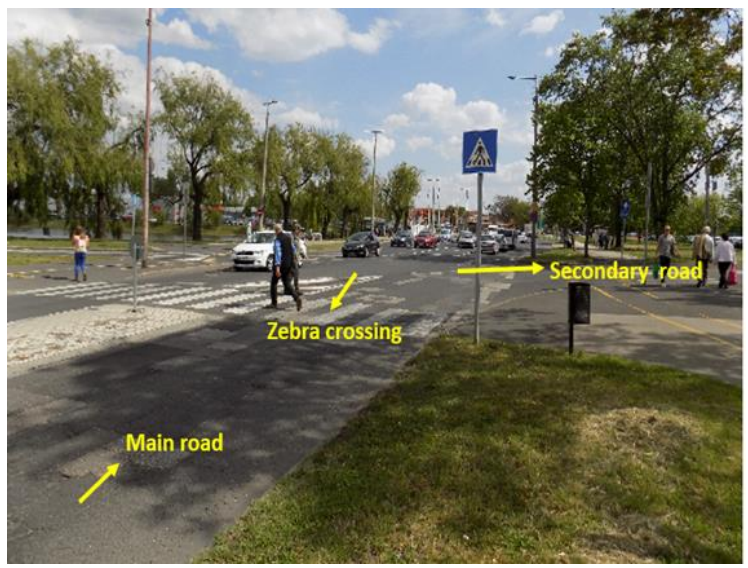

Figure 8. Tihanyi Árpád út 78, 9023 way - Jereváni way 5.3.3. Location (3): Ifjúság krt. 97, 9023- Kodály Zoltán

This location is the intersection of the main road (Ifjúság krt. 97, 9023) and the secondary road (Kodály Zoltán) (see Figs. 9 and 10). A zebra crossing exists on the main road. This site locates close to the previous locations but it is less crowded. The drivers use higher speeds on this main road compared to locations 1 and 2.

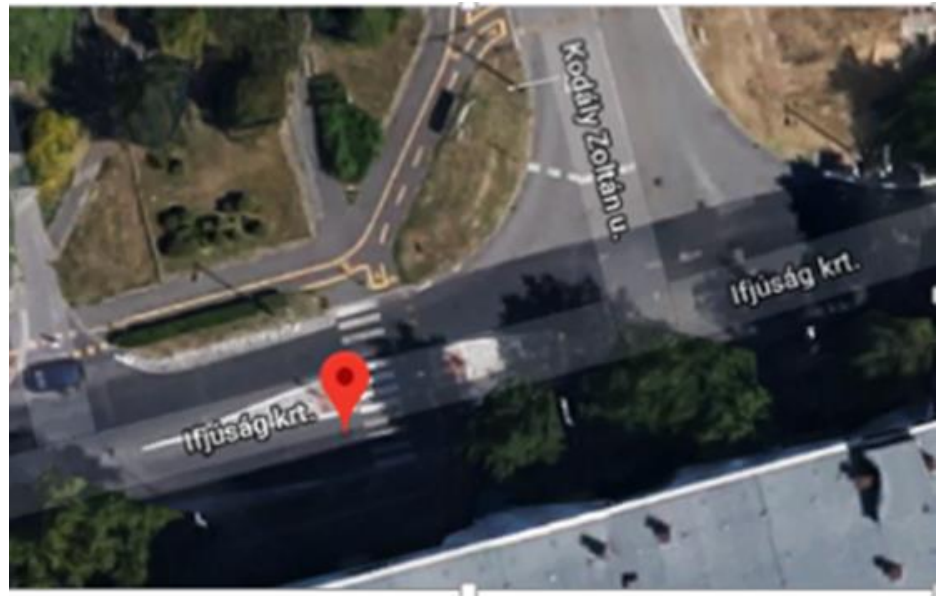

Figure 9. Ifjúság krt. 97, 9023 - Kodály Zoltán [16] 
Table 6. Some details related to location (3) (Ifjúság krt. 97, 9023 Kodály Zoltán) (based on [8] and [16])

\begin{tabular}{|c|c|c|c|}
\hline City & The main road & GPS Coordinates & $\begin{array}{c}\text { Number of } \\
\text { accidents } \\
\text { observed } \\
\text { according to } \\
\text { accidents map }\end{array}$ \\
\hline Győr & $\begin{array}{c}\text { Ifjúság krt. 97, } \\
9023\end{array}$ & $47^{\circ} 40^{\prime} 16.8^{\prime \prime} \mathrm{N}$ & Györ \\
\hline
\end{tabular}

Table 7. The specifications of recorded accidents at location (3) (Ifjúság krt. 97, 9023 - Kodály Zoltán) (based on [8] and [16])

\begin{tabular}{|c|c|c|c|c|}
\hline & $\begin{array}{c}\text { Time of } \\
\text { accident }\end{array}$ & $\begin{array}{c}\text { Outcome } \\
\text { of } \\
\text { accident }\end{array}$ & GPS & Accident Type \\
\hline $\begin{array}{c}\text { The } \\
\text { reported } \\
\text { accident }\end{array}$ & $9 / 1 / 2017$ & $\begin{array}{c}\text { serious } \\
\text { injury }\end{array}$ & $\begin{array}{c}47.671333 \\
17.654642\end{array}$ & $\begin{array}{c}\text { irregular movement at } \\
\text { the designated } \\
\text { pedestrian crossing }\end{array}$ \\
\hline
\end{tabular}

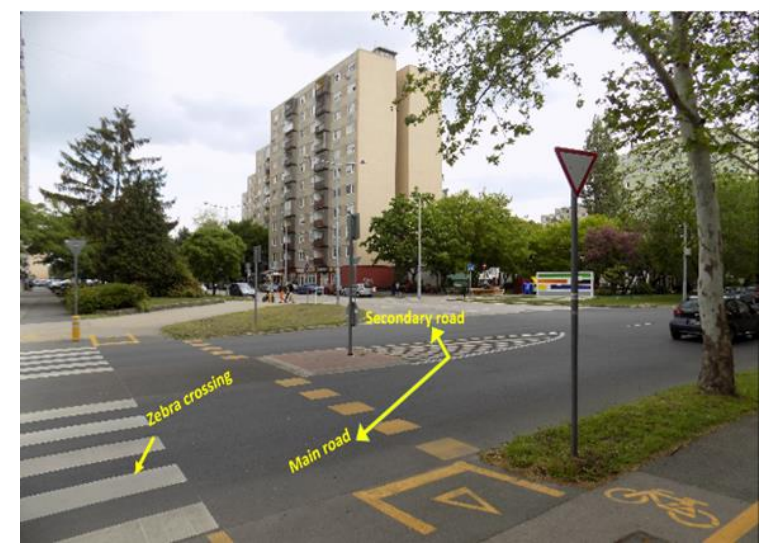

Figure 10.Ifjúság krt. 97, 9023- Kodály Zoltán 


\subsection{Data collection protocol}

The aim of study is to observe drivers, pedestrians and bicyclists behaviour at the pedestrian crossing and cycling lanes, and to apply the Swedish traffic conflict technology at each site to evaluate the severity of observed conflicts.

In this work, vehicle-pedestrian and bicyclist conflicts were selected to be observed. In case of bicyclists, it is much more common to swerve rather than brake (as an evasive action). As for pedestrians, they are able to stop or even jump back at literally no time. Moreover, in conflicts including pedestrians or bicyclists, they also very often become the relevant road users, i.e. take the first evasive action. As their speeds are generally lower compared to motor vehicles, the Conflict Speed value "pulls down" the conflict into lower severity. At least two potential solutions for this problem have been suggested: [10]

1. Svensson [17] suggested that the line dividing the serious and not serious conflicts should be moved one or two levels down (i.e. to the severity levels 25 or 24). This would include more conflicts into the final analysis and compensate for the under-scoring of the bicyclist/pedestrian conflicts.

2. Shbeeb [18] suggested that in the situations with pedestrian, it should always be the speed of the motor vehicle used as the Conflicting Speed, regardless to who is taking the evasive action. This is what was adopted in this study.

It was considered that the driver always takes the evasive action (braking). In this case, we estimate the driving speed as a conflicting speed, and the distance (between vehicle and pedestrian in case vehicle-pedestrian conflict observation, and between vehicle and bicyclist in case vehicle-bicyclist conflict observation).

The tasks as an observer on- site were as follows:

- detecting the conflict between the two road-users.

- filling in other relevant information together with a verbal description of the course of events.

- making a sketch of the conflict

- calculating Time-to-Accident (TA) values based on the conflicting speed and the distance between the two conflicting road-users for each conflict and then drop the data (conflicting speed and time-to-accident values for each conflict) on the Swedish conflict graph. This is to be made in both cases, vehicle-pedestrian conflicts and vehicle- bicyclist conflicts. 


\subsubsection{Equipment required for observation}

- Conflict register forms.

No specific form was adopted during observation period. The data were registered on a notebook, such as: the location, observations date and time, the two conflicting road users, the speed of vehicle when taking an evasive action (braking), the distance between the two confliction road users (the distance to the collision point), the conversion table (to convert speed and distance to time TA) shown in table (1), the calculated TA-value, a sketch of the conflict, and verbal description of the course of events,

We can also calculate the time as a distance divided by the speed by an equation in a form of excel-sheet for each location. (Especially for the speed values, which fall ranging between intervals that were not mentioned directly in the converting table).

- A watch.

- A pencil.

- A simple video camera works together with the field work.

\subsubsection{Considerations during observation period}

According to the literature review related to the Swedish technique, the number of days proposed for the observations and the periods of recording these observations during the day are determined based on the frequency of the expected conflicts at the given location. The expected frequency of conflicts is usually derived from previous experiences. According to e.g. Abdul Manan and Várhelyi [12] at sites with major road safety problems short observation periods can be done as the number of conflicts per time unit is still relatively high there. Observations are usually done in periods of $1-2$ hours with breaks in between for the observer to recover. [10]

Since the three selected sites are often located in the most crowded areas of the city (especially the first and second sites), and they are the same sites where at least an accident occurred in recent years between 2014 and 2018, the survey was conducted first in order to reveal the nature of traffic conflicts between road users personally through on-site visits, so that the measurements taken are objective as possible, and then field measurements and the necessary data (speeds and distances) were estimated. Even though the estimated measurements (in some cases) may not accurately reflect the real situation (the realistic values), but this approach may give at least a good background about the level of severity of the observed conflicts. 
Some other considerations during the observation:

- An observational point has selected at each location to offer a clear view over the observational area.

- The observer is good at driving process; speed estimations were not an issue.

- To facilitate estimation of distance and speed, initial measurements by the observer have done at the first arrival at the scene (distance between salient objects or marks can be measured).

- The same number of conflicts were considered for all locations. Once we got an equal number of conflicts at each location, the observation process stops. The field measurements ended within two weeks for the three locations.

- Three types of conflicts were adopted at each location. Six conflicts for each type, as will be illustrated in field measurement section.

\subsection{Field measurements}

Since many facilities exist near the three selected sites such as Fekete István Györ Primary School, Petz Aladár County Teaching Hospital, and Győr Pláza shopping Mall, the sites survey was comprehensive, several road users (motorists-pedestriansbicyclists) were frequently present and different kinds of conflicts could be observed. The results are represented in Figs. 11-16 and in Tables 8-10 (see Paragraphs 5.5.1-5.5.3).

\subsubsection{Location (1): Tihanyi Árpád út 73, 9023 - Kassák Lajos 73 St.}
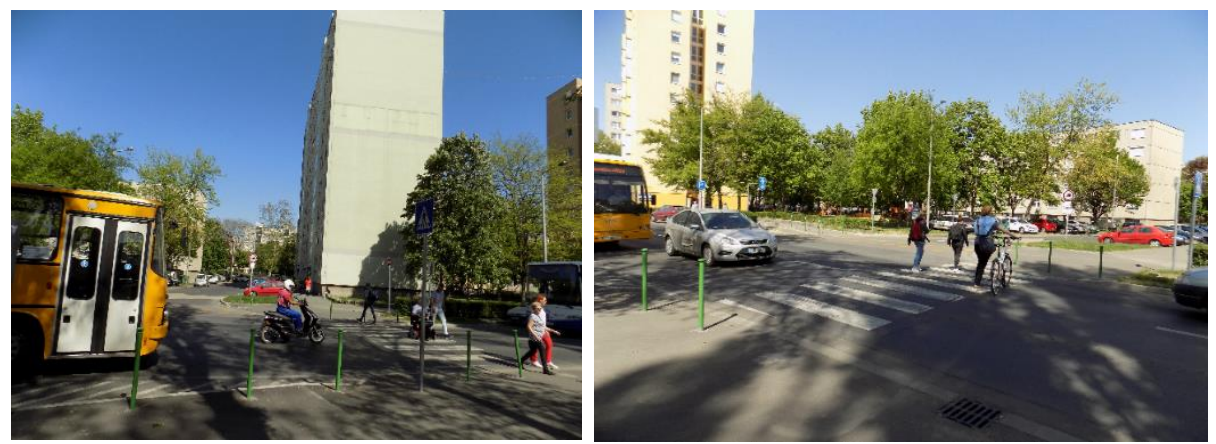

Figure 11. Photos taken for some conflicts observed at location (1) - Tihanyi Árpád út 73, 9023 - Kassák Lajos 73 St. 
A. Kizawi-Acta Technica Jaurinensis, Vol. 14, No. 4, pp. 377-405, 2021

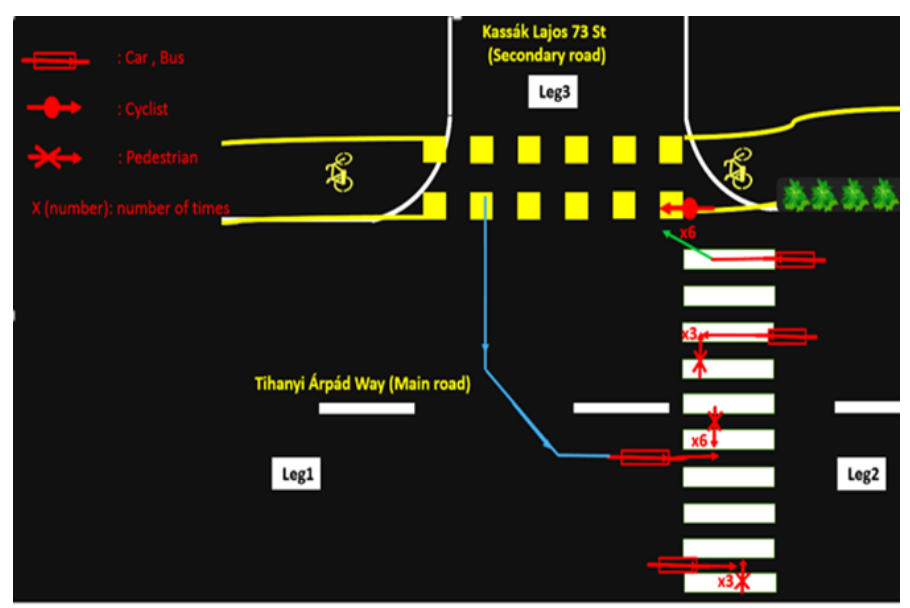

Figure 12. A sketch of the types of conflicts observed at location (1) - Tihanyi Árpád út 73, 9023 - Kassák Lajos 73 St. 
Table 8. Summary table of conflicts observation at location (1) - Tihanyi Árpád út 73, 9023 - Kassák Lajos 73 St.

\begin{tabular}{|c|c|c|c|c|c|c|c|}
\hline \multicolumn{8}{|c|}{ Mhin Road: Tihanyi Ár pádW ay. Secondary Road: Kassák Lajos 73 St. } \\
\hline Conflict Type & Road user 1 & Road us er 2 & $\begin{array}{l}\text { conflict } \\
\text { number }\end{array}$ & $\begin{array}{c}\text { Distance } \\
{[\mathrm{m}]}\end{array}$ & $\begin{array}{c}\text { Conflicting } \\
\text { Speed } \\
{[\mathrm{km} / \mathrm{hour}]}\end{array}$ & $\begin{array}{c}\text { Conflicting } \\
\text { Speed } \\
{[\mathrm{m} / \mathrm{S} \mathrm{ec}]}\end{array}$ & $\begin{array}{c}\text { Time-To- } \\
\text { Accident } \\
\text { [Sec] }\end{array}$ \\
\hline \multirow{6}{*}{$\begin{array}{c}\text { vehicle straight, } \\
\text { pedestrian }\end{array}$} & \multirow{6}{*}{ vehicle } & \multirow{6}{*}{ Pedestrian } & 1 & 3.5 & 30 & 8.3 & 0.42 \\
\hline & & & 2 & 3.5 & 32 & 8.9 & 0.39 \\
\hline & & & 3 & 3.5 & 30 & 8.3 & 0.42 \\
\hline & & & 4 & 4 & 35 & 9.7 & 0.41 \\
\hline & & & 5 & 3 & 30 & 8.3 & 0.36 \\
\hline & & & 6 & 4 & 32 & 8.9 & 0.45 \\
\hline \multirow{6}{*}{$\begin{array}{c}\text { vehicle turning left, } \\
\text { pedestrian }\end{array}$} & \multirow{6}{*}{ vehicle } & \multirow{6}{*}{ Pedestrian } & 1 & 3.5 & 20 & 5.6 & 0.63 \\
\hline & & & 2 & 3 & 22 & 6.1 & 0.49 \\
\hline & & & 3 & 3.5 & 18 & 5 & 0.7 \\
\hline & & & 4 & 3.5 & 20 & 5.6 & 0.63 \\
\hline & & & 5 & 2.5 & 18 & 5 & 0.5 \\
\hline & & & 6 & 3 & 20 & 5.6 & 0.54 \\
\hline \multirow{6}{*}{$\begin{array}{l}\text { vehicle turning } \\
\text { right, bicyclist }\end{array}$} & \multirow{6}{*}{ vehicle } & \multirow{6}{*}{ bicyclist } & 1 & 2.5 & 18 & 5 & 0.5 \\
\hline & & & 2 & 2.5 & 18 & 5 & 0.5 \\
\hline & & & 3 & 2.5 & 20 & 5.6 & 0.45 \\
\hline & & & 4 & 3.5 & 20 & 5.6 & 0.63 \\
\hline & & & 5 & 3 & 18 & 5 & 0.6 \\
\hline & & & 6 & 3.5 & 18 & 5 & 0.7 \\
\hline
\end{tabular}

5.5.2. Location (2): Tihanyi Árpád út 78, 9023 way - Jereváni way
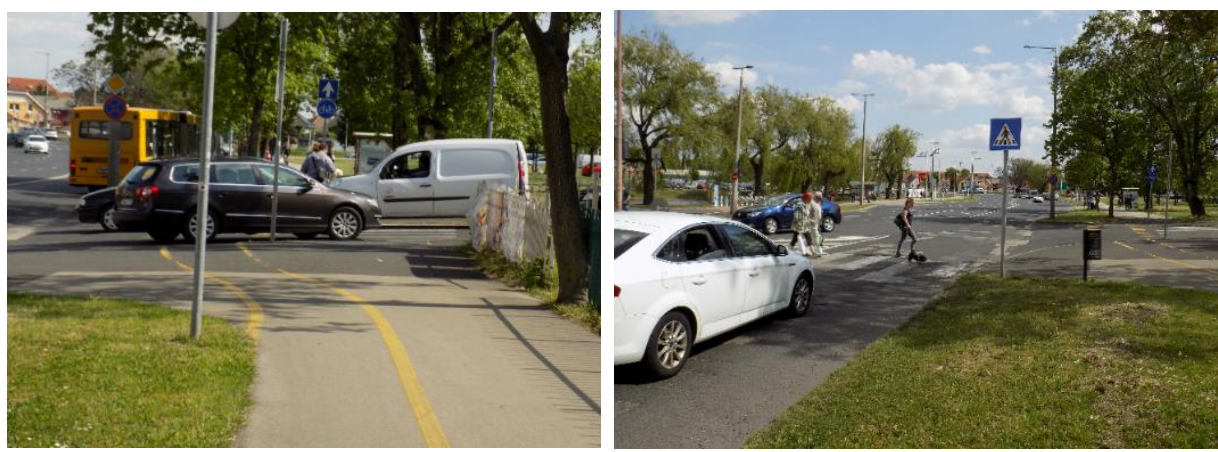

Figure 13. photos taken for some conflicts observed at location (2)-Tihanyi Árpád út 78, 9023 way - Jereváni way 


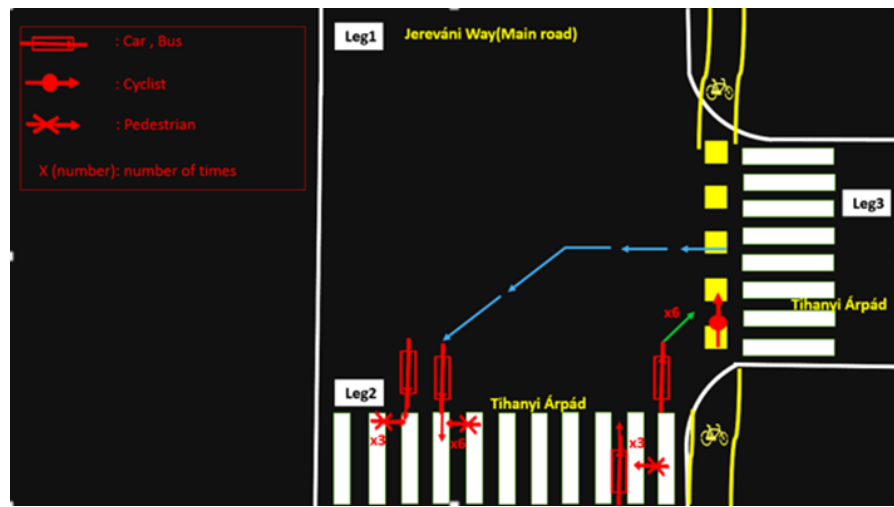

Figure 14. A sketch of the types of conflicts observed at location (2)-Tihanyi Árpád út 78, 9023 way-Jereváni way

Table 9. Summary table of conflicts observation at location (2) - Tihanyi Árpád út 78, 9023 way-Jereváni way

\begin{tabular}{|c|c|c|c|c|c|c|c|}
\hline \multicolumn{8}{|c|}{ Main Road: Tihanyi ÁrpádWay-Jer eváni Way. Secondary Road: Tihanyi Árpád. } \\
\hline Conflict Type & Road user 1 & Road user 2 & $\begin{array}{l}\text { conflict } \\
\text { number }\end{array}$ & $\begin{array}{c}\text { Distance } \\
{[\mathrm{m}]}\end{array}$ & $\begin{array}{c}\text { Conflicting } \\
\text { Speed } \\
{[\mathrm{km} / \text { hour }]}\end{array}$ & $\begin{array}{c}\text { Conflicting } \\
\text { Speed } \\
{[\mathrm{m} / \mathrm{Sec}]}\end{array}$ & $\begin{array}{c}\text { Time-To- } \\
\text { Accident } \\
\text { [Sec] }\end{array}$ \\
\hline \multirow{6}{*}{$\begin{array}{c}\text { vehicle straight, } \\
\text { pedestrian }\end{array}$} & \multirow{6}{*}{ vehicle } & \multirow{6}{*}{ Pedestrian } & 1 & 6 & 45 & 12.5 & 0.48 \\
\hline & & & 2 & 6 & 42 & 11.7 & 0.51 \\
\hline & & & 3 & 3.5 & 32 & 8.9 & 0.39 \\
\hline & & & 4 & 5.5 & 42 & 11.7 & 0.47 \\
\hline & & & 5 & 4 & 28 & 7.8 & 0.51 \\
\hline & & & 6 & 4 & 35 & 9.7 & 0.41 \\
\hline \multirow{6}{*}{$\begin{array}{c}\text { vehicle tuming left, } \\
\text { pedestrian }\end{array}$} & \multirow{6}{*}{ vehicle } & \multirow{6}{*}{ Pedestrian } & 1 & 4 & 28 & 7.8 & 0.51 \\
\hline & & & 2 & 3.5 & 28 & 7.8 & 0.45 \\
\hline & & & 3 & 4 & 25 & 6.9 & 0.58 \\
\hline & & & 4 & 2.5 & 22 & 6.1 & 0.41 \\
\hline & & & 5 & 3.5 & 22 & 6.1 & 0.57 \\
\hline & & & 6 & 3.5 & 22 & 6.1 & 0.57 \\
\hline \multirow{6}{*}{$\begin{array}{l}\text { vehicle turning } \\
\text { right, bicyclist }\end{array}$} & \multirow{6}{*}{ vehicle } & \multirow{6}{*}{ bicyclist } & 1 & 2 & 15 & 4.2 & 0.48 \\
\hline & & & 2 & 2.5 & 15 & 4.2 & 0.6 \\
\hline & & & 3 & 1.5 & 12 & 3.3 & 0.45 \\
\hline & & & 4 & 2 & 15 & 4.2 & 0.48 \\
\hline & & & 5 & 2.5 & 15 & 4.2 & 0.6 \\
\hline & & & 6 & 2.5 & 18 & 5 & 0.5 \\
\hline
\end{tabular}


5.5.3. Location (3): Ifjúság krt. 97, 9023 - Kodály Zoltán St.
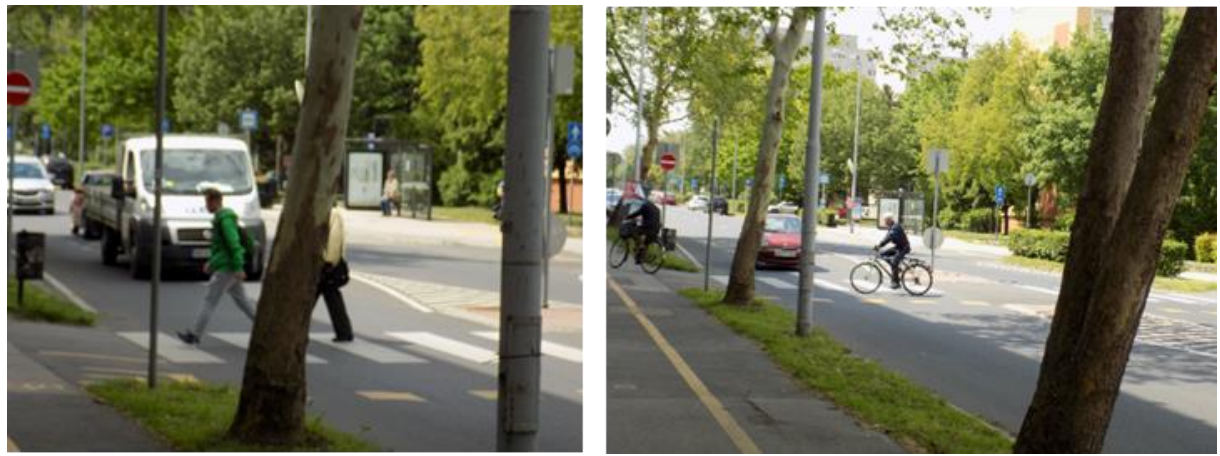

Figure 15. Photos taken for some conflicts observed at location (3) - Ifjúság krt. 97, 9023 - Kodály Zoltán St.

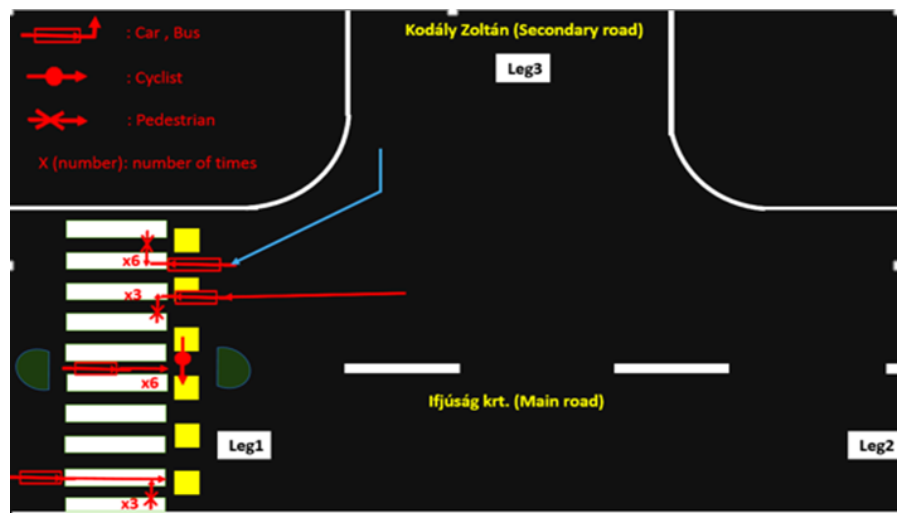

Figure 16. A sketch of the types of conflicts observed at location (3)-Ifjúság krt. 97, 9023 - Kodály Zoltán St. 
A. Kizawi-Acta Technica Jaurinensis, Vol. 14, No. 4, pp. 377-405, 2021

Table 10. Summary table of conflicts observation at location (3) - Ifjúság krt. 97, 9023 - Kodály Zoltán St.

\begin{tabular}{|c|c|c|c|c|c|c|c|}
\hline \multicolumn{8}{|c|}{ Main Road: Irjúság krt. Secondary Road: Kodály Zoltán St. } \\
\hline Conflict Type & Road user 1 & Road user 2 & $\begin{array}{l}\text { conflict } \\
\text { number }\end{array}$ & $\begin{array}{c}\text { Distance } \\
{[\mathrm{m}]}\end{array}$ & $\begin{array}{l}\text { Conflicting } \\
\text { Speed } \\
{[\mathrm{km} / \text { hour }]}\end{array}$ & $\begin{array}{c}\text { Conflicting } \\
\text { Speed } \\
{[\mathrm{m} / \mathrm{Sec}]}\end{array}$ & $\begin{array}{c}\text { Time-To- } \\
\text { Accident } \\
\text { [Sec] }\end{array}$ \\
\hline \multirow{6}{*}{$\begin{array}{c}\text { vehicle straight, } \\
\text { pedestrian }\end{array}$} & \multirow{6}{*}{ vehicle } & \multirow{6}{*}{ Pedestrian } & 1 & 6 & 42 & 11.7 & 0.51 \\
\hline & & & 2 & 5.5 & 38 & 10.6 & 0.52 \\
\hline & & & 3 & 5 & 35 & 9.7 & 0.51 \\
\hline & & & 4 & 3.5 & 32 & 8.9 & 0.39 \\
\hline & & & 5 & 4 & 32 & 8.9 & 0.45 \\
\hline & & & 6 & 5 & 38 & 10.6 & 0.47 \\
\hline \multirow{6}{*}{$\begin{array}{l}\text { vehicle turning } \\
\text { right, pedestrian }\end{array}$} & \multirow{6}{*}{ vehicle } & \multirow{6}{*}{ Pedestrian } & 1 & 4.5 & 20 & 5.6 & 0.81 \\
\hline & & & 2 & 3.5 & 22 & 6.1 & 0.57 \\
\hline & & & 3 & 4 & 20 & 5.6 & 0.72 \\
\hline & & & 4 & 4 & 22 & 6.1 & 0.65 \\
\hline & & & 5 & 3.5 & 22 & 6.1 & 0.57 \\
\hline & & & 6 & 4 & 25 & 6.9 & 0.58 \\
\hline \multirow{6}{*}{$\begin{array}{l}\text { vehicle straight, } \\
\text { bicyclist }\end{array}$} & \multirow{6}{*}{ vehicle } & \multirow{6}{*}{ bicyclist } & 1 & 5.5 & 40 & 11.1 & 0.5 \\
\hline & & & 2 & 4.5 & 35 & 9.7 & 0.46 \\
\hline & & & 3 & 5 & 38 & 10.6 & 0.47 \\
\hline & & & 4 & 4 & 32 & 8.9 & 0.45 \\
\hline & & & 5 & 3.5 & 28 & 7.8 & 0.45 \\
\hline & & & 6 & 4.5 & 30 & 8.3 & 0.54 \\
\hline
\end{tabular}

\section{Results and discussions}

The authors summarize their results in Figs. 17-19, see Paragraphs 6.1-6.3. The results are based on the author's literature review [19]. 


\subsection{Location (1): Tihanyi Árpád út 73, 9023 - Kassák Lajos 73 St.}

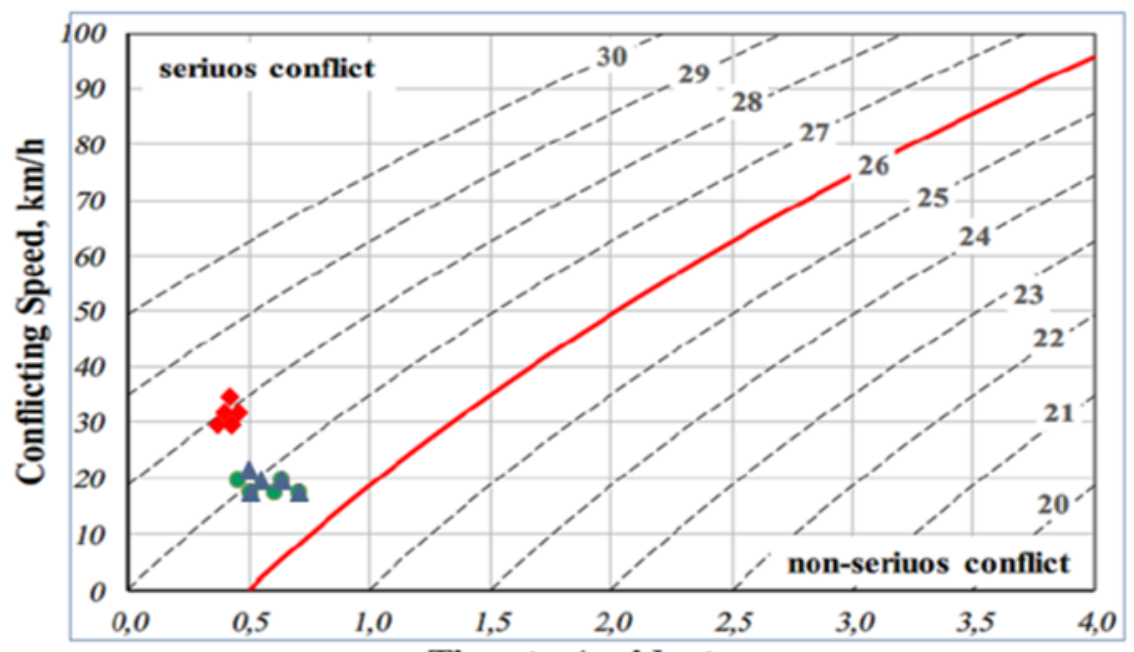

Time-to-Accident, sec.

Figure 17. The Swedish graph for location (1) - Tihanyi Árpád út 73, 9023 Kassák Lajos 73 St.

- The conflicts indicated by a rhombus: "vehicle move straight on the main road, pedestrian at zebra crossing"; Since this location is classified as one of the most crowded streets in the city, this causes a great potential of traffic conflicts occurrence between road users with a high frequency during the day. Pedestrianvehicle conflicts on the Main road fall around severity curve (No. 28) to be considered as the most serious conflicts compared to other types of conflict at this location.

- The conflicts indicated by a triangle: "vehicle on the secondary road turning left to the main, pedestrian at zebra crossing" and the conflicts indicated by a circle: "vehicle on the main turning right to the secondary, bicyclist at cycling lane"; The speeds at which the drivers began to take the evasive actions are almost identical for both types; the fact here is that when drivers change their direction from main to secondary or from secondary to main), they often try to keep attention to the traffic movements and move with lower speeds. Most conflicts fall within the 26-27 severity levels. They are classified within the serious conflict zone, but less severity than the first type. The values of time-to-accident are a bit higher than first type due to the lower speeds. 


\subsection{Location (2): Tihanyi Árpád út 78, 9023 way - Jereváni way}

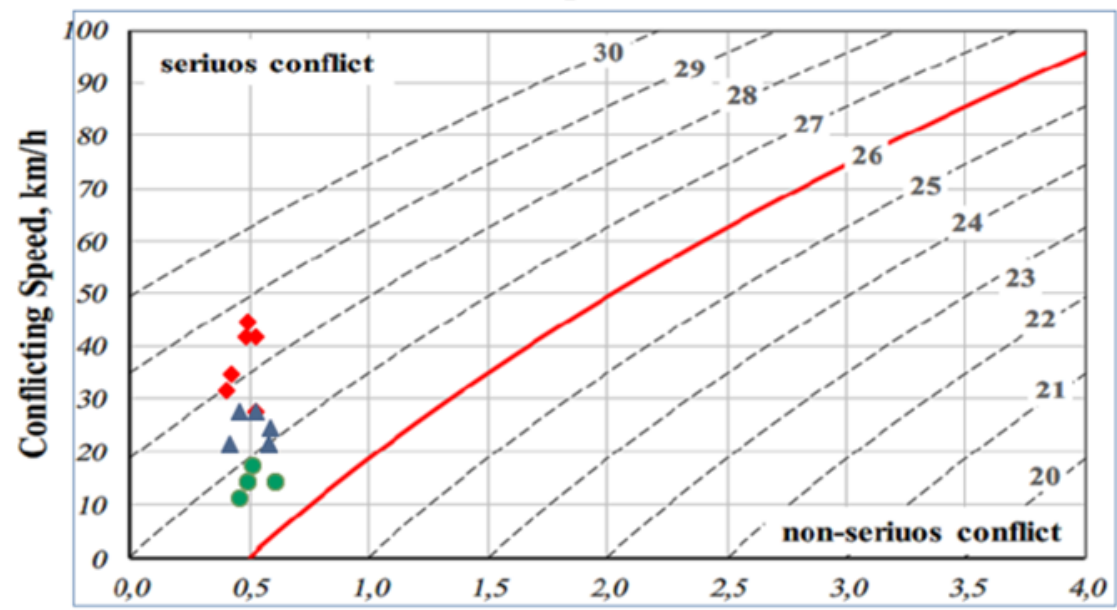

Time-to-Accident, sec.

Figure 18. The Swedish graph for location (2) - Tihanyi Árpád út 78, 9023 way Jereváni way

- The conflicts indicated by a rhombus: "vehicle move straight on the main, pedestrian at zebra crossing"; the speed values at which the drivers began to take the evasive action on the main road (Tihanyi Árpád Way-Jereváni Way) are higher than location (1). The drivers here have more freedom at driving speeds as they move (from Tihanyi Árpád Way) to the wider main road (Jereváni Way). However, they are obligated to slow down suddenly when they arrive the zebra crossing. Since the speeds are relatively higher, most of pedestrian-vehicle conflicts on the main road fall within the $28-29$ severity levels.

- The conflicts indicated by a triangle: "vehicle on the secondary turning left to the main, pedestrian at zebra crossing"; most conflicts of this type fall within the 2728 severity levels. The drivers who turn from the secondary road to Tihanyi Árpád way on their left are trying to accelerate a bit when they do not meet any traffic movements approaching to Jereváni Way, at this acceleration they may suddenly brake for a pedestrian at the zebra crossing.

- the conflicts indicated by a circle: "vehicle on the main turning right to the secondary, bicyclist at cycling lane "; the speeds at which the drivers began to take the evasive actions when turning from the main road to the secondary road are low, since the secondary road here is an entrance / exit for Györ Plaza shopping mall, and at this point they can only enter with low speeds. All conflicts 
of this type fall within the $26-27$ severity levels (the area close to the neutral line).

\subsection{Location (3): Ifjúság krt. 97, 9023 - Kodály Zoltán St.}

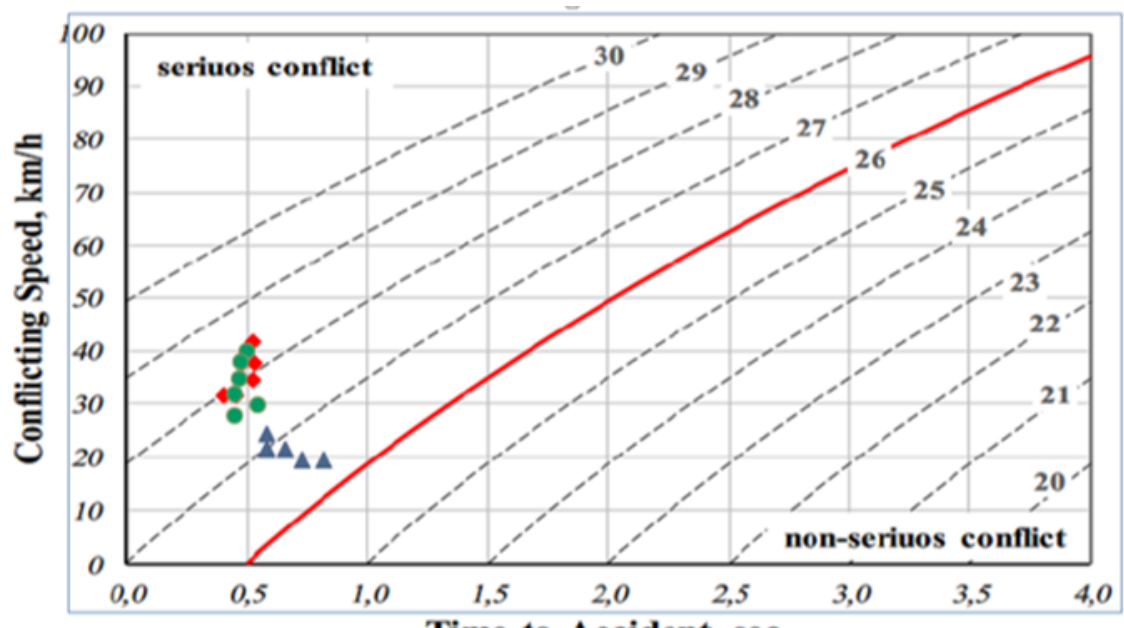

Time-to-Accident, sec.

Figure 19. The Swedish graph for location (3) - Ifjúság krt. 97, 9023 - Kodály Zoltán St.

- The conflicts indicated by a rhombus: "vehicle move straight on the main, pedestrian at zebra crossing"; this street is less crowded than the previous locations $(1,2)$. Even though the drivers are braking mostly at logical distances reaching to 6 meters, the situation is still dangerous since we are on a main road. We note that pedestrian-vehicle conflicts on the main road fall around severity curve (No. 28) and are considered the most dangerous conflicts.

- The conflicts indicated by a triangle: "vehicle on the secondary turning right to the main, pedestrian at zebra crossing"; most conflicts of this type fall within the 2627 severity levels. This type has less severity than the situation on the main road in the first type.

- The conflicts indicated by a circle: "vehicle move straight on the main, bicyclist at cycling lane "; we note that bicyclist -vehicle conflicts on the main road fall around severity curve (No. 28) and are considered the most dangerous conflicts. Most of time to accident values are less than 0.5 second. This indicates shorter time to collision. 


\section{Conclusions}

Accidents database and the technical approach for conflicts observation can be used as two methods to analyze traffic conflicts, as these two methods complement each other to gain more accurate results about the probability of accidents at a given location.

Looking at the results, and since the Swedish technique has been applied at the same locations where some accidents happened, we can say that there is a compatibility between Swedish technology and accident database regarding the seriousness of conflicts and probability of accidents.

Most pedestrian and bicyclist - vehicle conflicts are the most serious conflicts at all locations. The drivers on the main roads do not stop "in some cases" at zebra crossing for pedestrians as it was noticed during sites visit. Based on the findings, improving pedestrian accident countermeasures is always needed. The safety of vulnerable road users has to be always supported by implementation the traffic calming measures and accidents countermeasures especially at un-signalized intersections.

\section{References}

[1] A. Levy, In Canada, In Adoption \& Fostering 8 (1984) doi: https://doi.org/10.1177/030857598400800119

[2] Abhishek, M. Mandjes et al., Congestion analysis of unsignalized intersections (2018), ArXiv.

[3] S. M. Sohel Mahmud, L. Ferreira et al., Reviewing traffic conflict techniques for potential application to developing countries. Journal of Engineering Science and Technology 13 (6) (2018) pp. 1869-1890.

[4] G.B. Grayson, C. Hyden et al., The Malmo study, a calibration of traffic conflict techniques. A study organised by ICTCT - the International Committee on Traffic Conflict Techniques. Institute for Road Safety Research SWOV, The Netherlands (1984).

[5] C. Hydén, The development of a method for traffic safety evaluation: the Swedish traffic conflict technique. Doctoral thesis. Lund University, Department of Traffic Planning and Engineering, (1987). 
[6] S.Turner, K. Fitzpatrick et al., Motorist yielding to pedestrians at unsignalized intersections findings from a national study on improving pedestrian safety, Journal of the Transportation Research Board, No. 1982, Transportation Research Board of the National Academies, Washington, D.C., 2006, pp. 112.

doi: https://doi.org/10.3141/1982-03

[7] T.Q. Vuong, Traffic Conflict Technique Development for Traffic Safety Evaluation under Mixed Traffic Conditions of Developing Countries. Journal of Traffic and Transportation Engineering 5 (4) (2017) pp. 228-235. doi: https://doi.org/10.17265/2328-2142/2017.04.004

[8] WEB-BAL. Accident database management software (2018).

[9] R.S. Perkins, J.I. Harris, Traffic conflict characteristics - Accident potential at intersections. Highway Research Record No. 224 (1968), pp. 35-43.

[10] S. Almqvist, L. Ekman, The Swedish traffic conflict technique observer's manual. (July 2001), pp. 1-4.

[11] P. Zajíc, Traffic Conflicts and Road Transport Safety New Development (2012). Directive of the European Parliament and of the European Council No. 2008/96/EC on road infrastructure safety management. 4(VII).

[12] M. M. Abdul Manan, A. Várhelyi, Motorcyclists' road safety related behavior at access points on primary roads in Malaysia - A case study. Safety Science 77, (2015), pp. 80-94.

doi: https://doi.org/10.1016/j.ssci.2015.03.012

[13] S. Almqvist, C. Hyden, Methods for Assessing Traffic Safety in Developing Countries, Building Issues (LCHS) 6 (1) (1994) pp. 3-20. [cited 2021-03-15]. URL

http://www.Ith.se/fileadmin/hdm/BI_Volume_06_1_1994_Metho ds_for_Assessing_Traffic_Safety_in_Develop̄ment_-Countries. $p \overline{d f}$

[14] C. Hydén, L. Linderholm, The Swedish Traffic-Conflicts Technique. International Calibration Study of Traffic Conflict Techniques (1984) pp. 133-139.

doi: https://doi.org/10.1007/978-3-642-82109-7_12 
[15] G. Tiwari, D. Mohan, J. Fazio, Conflict analysis for prediction of fatal crash locations in mixed traffic streams. Accident Analysis \& Prevention 30 (2) (1998) pp. 207-215.

[16] Google Maps (2021) [cited 2021-03-15].

URL maps.google.com

[17] Å. Svensson, A method for analysing the traffic process in a safety perspective. Doctoral thesis. University of Lund, Lund Institute of Technology, Department of Traffic Planning and Engineering, (1998).

[18] L. Shbeeb, Development of a traffic conflicts technique for different environments - a comparative study of pedestrian conflicts in Sweden and Jordan. Doctoral thesis. University of Lund, Lund Institute of Technology, Department of Technology and Society, Traffic Engineering, (2000).

[19] A. Kizawi, A. Borsos, A Literature review on the conflict analysis of vehiclepedestrian interactions. Acta Technica Jaurinensis (2021). doi: https://doi.org/10.14513/actatechjaur.00601 Creative Commons Attribution NonCommercial ( $C C B Y-N C$ 4.0) license. 